\title{
A Comparative Study of the Pliability of Separate Auxetic Architectonic Structures by Means of CAD
}

\author{
Mª Dolores Álvarez Elipe \\ CEIPSO Salvador Dalí, Calle Tía Javiera, 6, 28942 Fuenlabrada, Madrid, Spain \\ E-mail: mdolores_500@hotmail.com
}

Received: 25 March 2020; Accepted: 9 April 2020; Available online: 25 June 2020

\begin{abstract}
If a lengthened structure gets fat in all directions and the same structure gets narrower when it is pressed, it is an auxetic structure. This happens because these kinds of structures have a negative Poisson's ratio (NPR). In this paper a comparative study of unity patterns of CAD auxetic geometries is presented. A Computer Aided Design library of auxetic geometries will be developed to apply them to architecture. The geometric behavior of the eighteen auxetic separate patterns is tested from the developed library in order to develop a systematic comparison, analyzing properties of these forms, such as their maximum achievable area reductions in relation with the total length of bars of the structure, in order to obtain a growth factor.
\end{abstract}

Keywords: Auxetic; Architecture; Geometries; Patterns; Growth.

\section{Introduction}

If a material is stretched, it usually thins. The Poisson ratio, $v=-d \varepsilon \operatorname{trans} / d \varepsilon a x i a l, \varepsilon t$ trans and $\varepsilon a x i a l$, can define numerically this change of dimensions, which are the transverse and axial deformations when the material expands or contracts in the axial direction. Generally speaking, $v_{i j}$ is the Poisson ratio that indicates a compression in the ' $j$ ' direction when a stretching is applied in the 'i' direction. Typical structures have a positive Poisson's ratio and it indicates that the material preserves its volume. If a lengthened structure gets fat in all directions and the same structure gets narrower when it is pressed, it is an auxetic structure. This happens because these kinds of structures have a negative Poisson's ratio (NPR) [1-4]. The auxetic properties of natural materials (skins, some minerals ...) and artificial materials (Gore-Tex ${ }^{\circledR}$, foams, polymeric foams) have been described. Molecular auxetic patterns to generate auxetic structures have been developed by many researches [5].

New products have been developed by the use of auxetic designs. The principal fields of use are morphological structures of forms, intelligent expandable actuators and minimally invasive implantable devices [6]. Shape memory auxetics alloys (SMA) used for the development of drop-down satellite antennas exist also [7]. They are used like smart actuators based on auxetic structures. Several stages of post-processing were iniciated in some investigations about the behavior of the shape memory polyurethane foams with auxetic properties [8]. In the matter of medical devices, the properties of some auxetic designs of novel proposals have evaluated to develop expandable stents [9]. The use of these materials and the behavior of their geometry in architecture and in the size of these, are not known at the moment. That is why auxetic materials can become novel models which will identify the most relevant properties for designing tasks of the most innovative variable geometries, which will be applied to the construction of new transformable architectures.

Some auxetic and potentially auxetic geometries, usually classified as "reentrant" [10], "chiral" and "rotating" because of the characteristics that generate the auxetic behavior, have been explained in previous reviews and investigations. However, information at the architectural scale is not provided. Sometimes, at the nanoscale, when subjected to uniaxial stresses, only a schematic of its folding process is delivered, which is scarce for later investigation studies [11]. The added investigation of the other properties of some auxetic geometry, systematic and comparative, would consequently be relevant to design new transformable structures for architecture.

In this paper a comparison of CAD unity patterns of auxetic geometries is presented. The beginning of the presented investigation focuses on the generation of some auxetic geometries developed by means of Computer Aided Design of 18 separate models of architecture, adapted or based on the information of previous papers, patents and conference, and some we have developed ourselves.

The models that are in nature or have been designed by other authors in scales different to architecture have rigid knots, but the present paper studies the behavior when these geometries have articulated knots. So, the results are totally independent of the materials. It is a theoretical study useful to know the capacity of folding in structures than can be used in architecture. 
When the CAD library is developed, the properties of some auxetic geometries were checked and a systematic comparison was developed, studying the special properties of those geometries for the generation of deployable structures for architecture, such as maximum area reductions (in relation with the total length of the bars of the structure), in order to obtain a growth factor.

As far we know, this investigation is the only exhaustive study of comparison of auxetic geometries in architecture done so far. The geometric variations on these models could be infinite, since we could verify multitude of behaviors varying the size of each bar of the separate structure. However, this study of geometric variations for the same pattern will not be developed. A comparison between regular separate patterns will be carried out.

\section{Methods and materials}

Some geometric patterns are developed using Autocad, by obtaining different cells of units. Possible positions of different auxetic typologies have been modeled in the space since they are completely folded until they reach their maximum opening, in order to analyze the relationship between the amount of used mass and the surface achieved. For this, we will count the total length of bars used in the design of each model, as an reference to the quantity of mass [12].

The goal is to obtain the lightness of auxetic structures and their capacity of pliability in order to make dropdown structures. The most important thing is the relation between full spaces and empty spaces. Full spaces are the bars, this is, everything that has some mass. Empty space is the volume of the structure, all that doesn't have mass. This is a theoretical study, because the bars in the reality have material, but this will be considered for future investigations. In the present study the focus will only be on the relation between quantity of bars and volume when the nodes are totally articulated.

In this study we will look for a general behavior in which the length indicates the linear structural elements. The relation of area $(A)$ and length $(\mathrm{L})$ will give us a ratio $(\mathrm{K})$, in order to know the growth relation of these kinds of structures.

Each design has and area, which will correspond to the polygon, circumference or square, (as appropriate), in which we inscribe the design. From the division and the subtraction of $K_{\max }$ and $K_{\min }$, FC (:) and FC (-) we obtain the growth ratios of each design [12]. The applicable patterns are studied separately in order to establish structural developments according to the size, as well as transforming architectures that follow novel geometric developments in deployable auxetic architectures. The studied structures have similar overall size, but some variances appear between them. That is because of the use of cell units with different degrees of complexity (see table 1).

These structures are: hexagonal re-entrant structure [13-17], star re-entrant structure [16], triangular re-entrant structure [18], square re-entrant structure, hexagonal honeycomb re-entrant structure [4], square grid re-entrant structure [4], lozenge grid re-entrant structure [19], triangular, square, rectangular, hexagonal and circular chiral structures [20], triangular, square, rectangular and different rectangles rotating units [21], square and rectangular micro-porous polymers [4].

The maximum area reduction achievable by each of the structures studied here is obtained by the limit cases, which help us in design jobs related with deployable architectures. Table 1 contains some draws of 2D possible auxetic separate structures for giving the deployable development applied in the evaluation of the proposed geometries-structures under study.

\section{Discussion and results}

\subsection{Auxetic geometries computer aided design library}

The CAD development of auxetic and potentially auxetic patterns in order to study them is summarized in Table 1 , which shows respectively the different CAD patterns of separate auxetic generated patterns. The majority of these names and geometries are picked from preceding publications, patents, websites and researches, and some of them present significant modifications or even our own new designs.

\subsection{Comparative study of the separate auxetic structures}

A total of 18 separate auxetic structures have been modeled in order to obtain detailed information such as maximum area reductions attainable in relation to the total length of bars of the structure, in order to obtain a growth factor. Table 1 indicates the behavior of these geometries, in order to use them as parts of a structure. The results of growth factor (FC (:) and FC (-)) of the separate auxetic geometries have been normalized, by dividing area (A) and length (L). 
Table 1. Deployable development of auxetic separate patterns, own elaboration

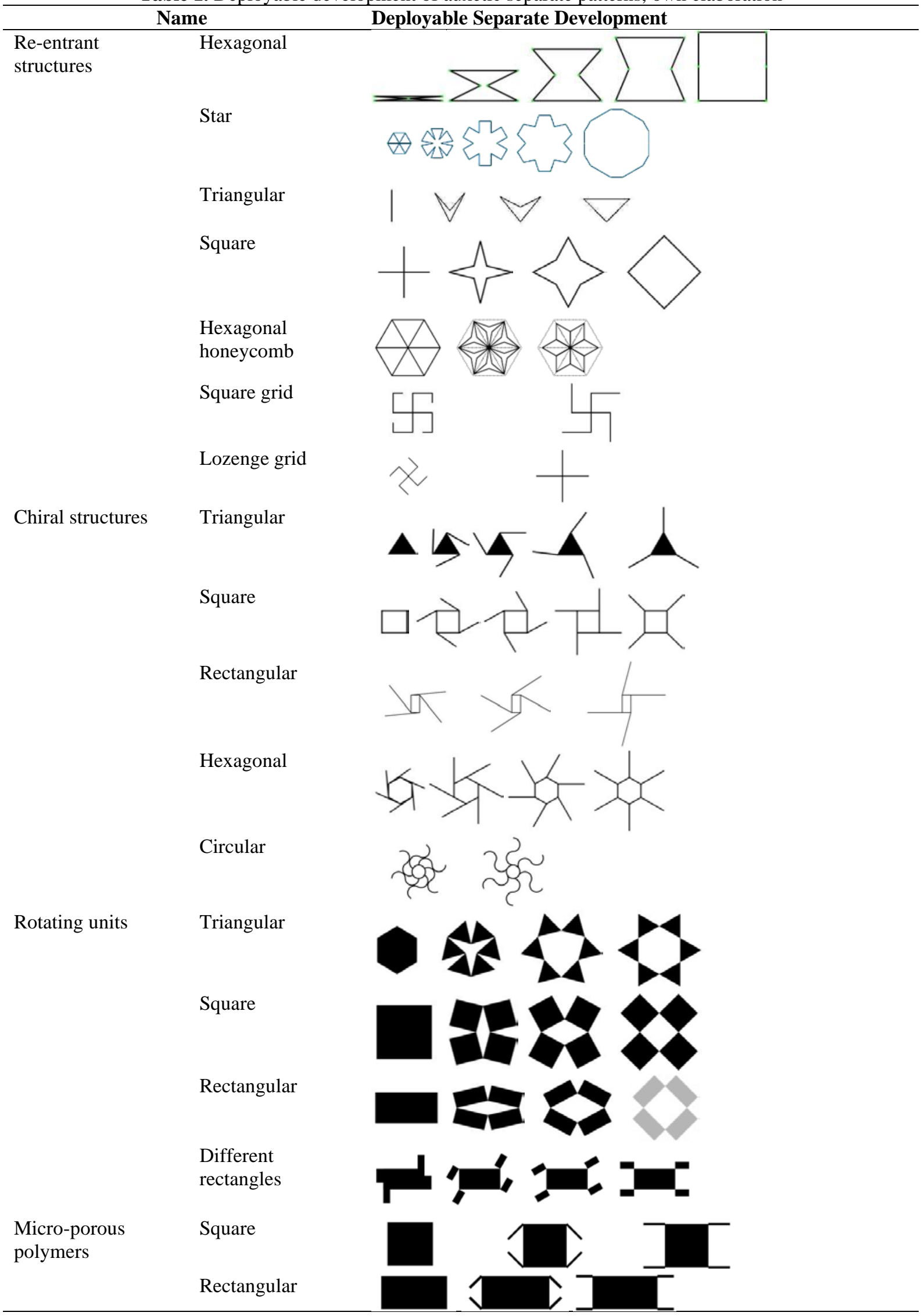

*Note: The rigid nucleus of the chiral structures, rotating units and micro-porous polymers can be full or without any mass. 


\subsection{Final discussion and summary}

A discussion of results is included in table 1, in which appear the relevant characteristics for 2D separate auxetic structures under study. From these results (see table 1) it is important to establish general trends and connections between the different properties of separate auxetic geometries. It is important to comment on some highly interesting details. The table that contains the values to study the geometric behavior of the separate auxetic structures is presented below (table 2), in which:

$\mathrm{L}$, Total length of bars in the considered structure

$\mathrm{V}_{\text {min }}$, Minimum volume in the considered structure

$\mathrm{V}_{\max }$, Maximum volume in the considered structure

$\mathrm{K}_{\min }, \mathrm{V}_{\min } / \mathrm{L}$ minimum ratio

$\mathrm{K}_{\max }, \mathrm{V}_{\max } / \mathrm{L}$ maximum ratio

FC (:), $K_{\max } / K_{\min }$ growth factor

FC (-), $K_{\max }-K_{\min }$ growth factor

M.P., Micro-porous polymers

From Table 2, we can create Figures 1 and 2, in which we can see, in a more visual way, the behavior of the separate auxetic structures, by FC (:) and FC (-).

It can be seen that the star-shaped reentrant design has a growth ratio much larger than the rest of the structures, so it would be very interesting to apply it as a deployable auxetic structure. But it is one of the structures less folding in the auxetic form, what it means a limitation (Table 2 and Figures 1 and 2). It makes necessary to have joints in a way that allows turns which generate non-auxetic folding. Like most separate reentrant structures, it consists of its joints in a single plane of several rods.

Table 2. geometric behavior of auxetic separate patterns, own elaboration

\begin{tabular}{|c|c|c|c|c|c|c|c|c|}
\hline Name & & $\mathbf{L}$ & $\mathbf{A}_{\min }$ & $\mathbf{A}_{\max }$ & $\mathbf{K}_{\min }$ & $\mathbf{K}_{\max }$ & FC (:) & FC (-) \\
\hline \multirow[t]{7}{*}{ Re-entrant structures } & Hexagonal & 4,00 & 0,00 & 1,00 & 0,00 & 0,25 & $\#_{\mathrm{j}} \mathrm{DIV} / 0$ ! & 0,25 \\
\hline & Star & 18,00 & 2,60 & 24,99 & 0,14 & 1,39 & 9,61 & 1,24 \\
\hline & Triangular & 4,00 & 0,00 & 0,43 & 0,00 & 0,14 & $\#_{\mathrm{j} D I V} / 0$ ! & 0,14 \\
\hline & Square & 8,00 & 2,00 & 4,00 & 0,25 & 0,50 & 2,00 & 0,25 \\
\hline & Hexagonal honeycomb & 18,00 & 1,82 & 2,60 & 0,10 & 0,14 & 1,43 & 0,04 \\
\hline & Square grid & 10,00 & 4,00 & 6,50 & 0,40 & 0,65 & 1,63 & 0,25 \\
\hline & Lozenge grid & 8,00 & 4,00 & 8,00 & 0,50 & 1,00 & 2,00 & 0,50 \\
\hline \multirow[t]{5}{*}{ Chiral structures } & Triangular & 6,00 & 0,43 & 3,23 & 0,07 & 0,54 & 7,51 & 0,47 \\
\hline & Square & 8,00 & 1,00 & 5,83 & 0,13 & 0,73 & 5,83 & 0,60 \\
\hline & Rectangular & 12,00 & 2,00 & 11,00 & 0,17 & 0,92 & 5,50 & 0,75 \\
\hline & Hexagonal & 12,00 & 2,60 & 10,39 & 0,22 & 0,87 & 4,00 & 0,65 \\
\hline & Circular & 25,13 & 18,31 & 28,27 & 0,73 & 1,12 & 1,54 & 0,40 \\
\hline \multirow[t]{4}{*}{ Rotating units } & Triangular & 18,00 & 2,60 & 7,79 & 0,14 & 0,43 & 3,00 & 0,29 \\
\hline & Square & 16,00 & 4,00 & 7,00 & 0,25 & 0,44 & 1,75 & 0,19 \\
\hline & Rectangular & 24,00 & 12,00 & 13,00 & 0,50 & 0,54 & 1,08 & 0,04 \\
\hline & Different rectangles & 42,00 & 41,00 & 50,00 & 0,98 & 1,19 & 1,22 & 0,21 \\
\hline \multirow[t]{2}{*}{ M.P. } & Square & 6,00 & 1,00 & 2,00 & 0,17 & 0,33 & 2,00 & 0,17 \\
\hline & Rectangular & 8,00 & 2,00 & 3,00 & 0,25 & 0,38 & 1,50 & 0,13 \\
\hline
\end{tabular}

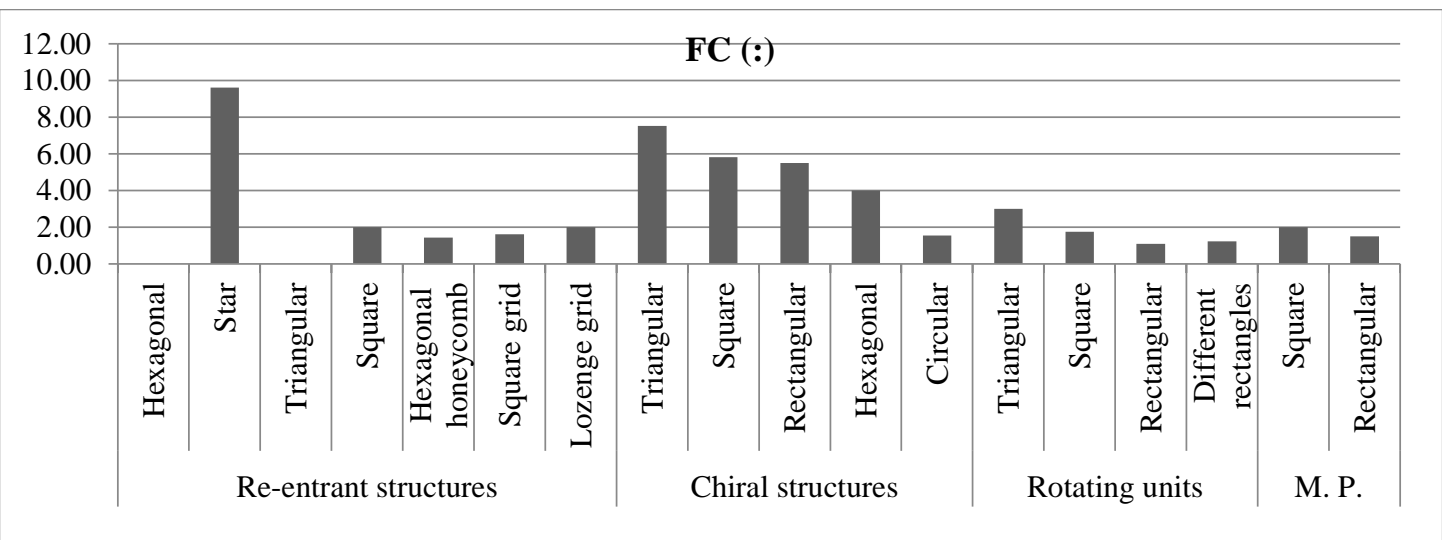

Figure 1. Comparison between growth factors of auxetic separate reentrant structures. They have been generated by division FC (:). Own elaboration. 


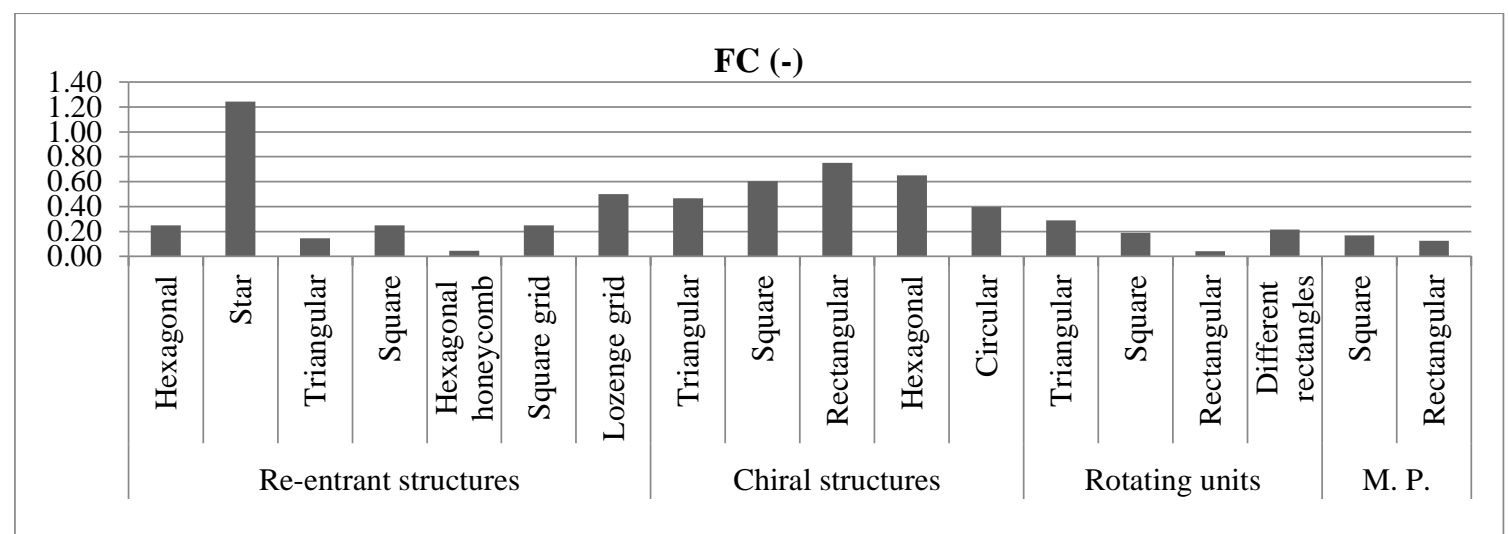

Figure 2. Comparison between growth factors of auxetic separate reentrant structures. They have been generated by subtraction FC (-). Own elaboration.

The turns that allow these joints go from $0^{\circ}$ to $150^{\circ}$. At first the apparent solution would be to give the articulation more breadth of rotation. However, if we study the geometry, the only form of non-auxetic folding for total packing of the structure is to develop an intermediate joint in the bars remaining on the outside after obtaining maximum auxetic folding. In addition, it would be necessary to introduce an opening system that allows opening this auxetic geometry to obtain its folding and packaging suitable for transport.

On the other hand, overall growth factors for separate chiral structures are observed. This type of designs only can pliable around the polygon in which the bars rotate. If we wanted more quantity of compacting, as in the preceding design, we would have to use a non-auxetic end fold (and this without mass inside the principal polygon). The non-auxetic pliability in chiral structures is easier to obtain. It consists of giving more turning angle to the joints by opening of one of the vertices of the polygon. In this way some bars can be folded over others [12].

It should also be noted that the separate hexagonal reentrant, triangular reentrant and tetrahedral reentrant structures are completely folded auxetically. It is a very useful aspect when we work with these designs to pliable architectures.

A CAD development of some auxetic geometries is provided (re-entrant, chiral, rotating..., all of which are separate planar structures) and awarded their typical values such as maximum area reductions attainable in relation with the total length of bars of the structure, in order to obtain a growth factor. We also have tried to check some known 'auxetic' designs, whose behavior is at the moment not auxetic when they are separate patterns, like triangular reentrant structure and rectangular rotating unit (only in its maximum aperture, in grey).

The provided values for the diverse characteristics can be interpreted as a previous award for all of the reviewed geometries, to assist in the tasks of choose some of them for architecture. When a structure is chosen for its growth factor, the specific effects of the geometric changes must be addressed, in case the adaptation of the final property is necessary for a specific application.

Interesting new designs, generated by modifications of some auxetic revisions, improve folding when the structure is compressed, reducing its volume. The structures also have minor stress efforts [13] [14]. The geometries of this library can be modified in order to obtain more possibilities of design. The combination of separate auxetic structures can be used to make other 2D or 3D auxetic designs. Respecting subsequent work pathways, it is useful providing other investigation centered on auxetic geometries based on a combination of a set of separate auxetic patterns, which helps progressively to improve the present CAD development of auxetic geometries.

\section{Conclusions}

It has been designed 18 separate auxetic structures in order to obtain relevant design properties, like maximum area reductions attainable in relation with the total length of bars of the structure, in order to obtain a growth factor. The auxetic characteristics of 18 auxetic structures have been validated by CAD simulation results. The set of separate auxetic structures and the generated conclusions may be useful for the emergence of new architectures with these special features. The separate non-auxetic structures can became auxetic through combination. That is how they may be used in pliable architectures, although the separate structure cannot be pliable.

\section{References}

[1] Lakes R. Foam structures with a negative Poisson's ratio. Science. 1987;235:1038-1040.

[2] Evans KE. Auxetic polymers: a new range of materials. Endeavour. 1991;15(4):170-174. 
[3] He C, Liu P, McMullan PJ, Griffin AC. Toward molecular auxetics: Main chain liquid crystalline polymers consisting of laterally attached para-quaterphenyls. Physica Status Solidi (B). 2005;242(3):576-584.

[4] Liu Y, Hu H. A review on auxetic structures and polymeric materials. Scientific Research and Essays. 2010;5(10):1052-1063.

[5] Griffin AC, Kumar S, Mc Mullan PJ. Textile fibers engineered from molecular auxetic polymers. National Textile Center Research Briefs-Materials Competency. 2005: 1-2.

[6] Álvarez MD. Tensioned auxetic structures manual calculus. Journal of Architectural Environment \& Structural Engineering. 2019;02(01):23-31.

[7] Scarpa F, Jacobs S, Coconnier C, Toso M, Di Maio D. Auxetic shape memory alloy cellular structures for deployable satellite antennas: design, manufacture and testing. In: EPJ Web of Conferences (Vol. 6). EDP Sciences. 2010.p. 27001.

[8] Bianchi M, Scarpa F, Smith CW. Shape memory behaviour in auxetic foams: mechanical properties. Acta Materialia. 2010;58(3):858-865.

[9] Tan TW, Douglas GR, Bond T, Phani AS. Compliance and longitudinal strain of cardiovascular stents: influence of cell geometry. Journal of Medical Devices. 2011;5(4): 041002.

[10] Friis EA, Lakes RS, Park JB. Negative Poisson's ratio polymeric and metallic foams. Journal of Materials Science. 1988;23(12):4406-4414.

[11] Álvarez JC, Díaz A. Comparative study of auxetic geometries by means of computer-aided design and engineering. Smart Materials and Structures. 2012;21(10):105004.

[12] Álvarez MD. Applications of the auxetic properties in architecture. [Doctoral Thesis]. E.T.S. Arquitectura (UPM). 2017. p. 86-185. (in Spanish)

[13] Mehta V, Frecker M, Lesieutre G. Contact-aided compliant mechanisms for morphing aircraft skin. In: Modeling, Signal Processing, and Control for Smart Structures (Vol. 6926,). International Society for Optics and Photonics. 2008. p. 69260C.

[14] Mehta V, Frecker M, Lesieutre GA. Stress relief in contact-aided compliant cellular mechanisms. Journal of Mechanical Design. 2009; 131(9): 091009.

[15] Goldstein RV, Gorodtsov VA, Lisovenko DS. Anomalous elastic behaviour of micro and nanowhiskers with a cubic atomic structure. Ishlinsky Institute for Problems in Mechanics RAS-Teaching material (Moscow: Russian Academy of Sciences).2010.

[16] Wu H, Wei,G. An approximation method for simulating temperature dependence of Poisson's ratios of selfexpanding auxegens. Comput. Methods Sci. Technol. 2004; 10(2):229-234.

[17] Grima JN, Gatt R, Alderson A, Evans KE. On the potential of connected stars as auxetic systems. Molecular Simulation. 2005;31(13):925-935.

[18] Ugbolue SC, Kim YK, Warner SB, Fan Q, Yang CL, Kyzymchuk O. Auxetic fabric structures and related fabrication methods. US Patent Specification 2011/0046715 A1. 2011.

[19] Larsen UD, Signund O, Bouwsta S. Design and fabrication of compliant micromechanisms and structures with negative Poisson's ratio. Journal of Microelectromechanical Systems. 1997;6(2):99-106.

[20] Aldred P, Moratti SC. Dynamic simulations of potentially auxetic liquid-crystalline polymers incorporating swivelling mesogens. Molecular Simulation. 2005;31(13):883-887.

[21] Dirrenberger J, Forest S, Jeulin D, Colin C. Homogenization of periodic auxetic materials. Proc. Eng. 2011; 10:1847-1852.

(C) 2020 by the author(s). This work is licensed under a Creative Commons Attribution 4.0 International License (http://creativecommons.org/licenses/by/4.0/). Authors retain copyright of their work, with first publication rights granted to Tech Reviews Ltd. 